\title{
Convergence Analysis of Iterative Sequences for a Pair of Mappings in Banach Spaces
}

\author{
L. C. Zeng ${ }^{1}$, N. C. Wong ${ }^{2}$, and J. C. Yao ${ }^{3}$
}

Communicated by Bingren Li

\begin{abstract}
Let $C$ be a nonempty closed convex subset of a real Banach space $E$. Let $S: C \rightarrow$ $C$ be a quasi-nonexpansive mapping, let $T: C \rightarrow C$ be an asymptotically demicontractive and uniformly Lipschitzian mapping, and let $F:=\{x \in C: S x=x$ and $T x=x\} \neq \emptyset$. Let $\left\{x_{n}\right\}_{n \geq 0}$ be the sequence generated from an arbitrary $x_{0} \in C$ by

$$
x_{n+1}=\left(1-c_{n}\right) S x_{n}+c_{n} T^{n} x_{n}, \quad n \geq 0 .
$$

We prove necessary and sufficient conditions for the strong convergence of the iterative sequence $\left\{x_{n}\right\}$ to an element of $F$. These extend and improve recent results of Moore and Nnoli.
\end{abstract}

Keywords: Quasi-nonexpansive mapping, asymptotically demicontractive type mapping, iterative sequence, convergence analysis.

2000 Mathematics Subject Classification: 47H09, 47H10, 47H17

1. Professor, Department of Mathematics, Shanghai Normal University, Shanghai, China. This research was partially supported by the Teaching and Research Award Fund for Outstanding Young Teachers in Higher Education Institutions of MOE, China and the Dawn Program Foundation in Shanghai. E-mail: zenglc@hotmail.com

2, 3. Professors, Department of Applied Mathematics, National Sun Yat-Sen University, Kaohsiung, Taiwan 80424. This research was partially supported by grant from the National Science Council of Taiwan. E-mail: wong@math.nsysu.edu.tw,yaojc@math.nsysu.edu.tw 


\section{Introduction}

Let $E$ be a real normed linear space. Let $\langle\cdot, \cdot\rangle$ denote the generalized duality pairing between $E$ and its dual space $E^{*}$. Let $J: E \rightarrow 2^{E^{*}}$ be the normalized duality mapping defined for each $x \in E$ by

$$
J(x)=\left\{f^{*} \in E^{*}:\left\langle x, f^{*}\right\rangle=\|x\|^{2}=\left\|f^{*}\right\|^{2}\right\} .
$$

It is well known that if $E^{*}$ is strictly convex then $J$ is single-valued. In the sequel we shall write $j$ for a (single-valued) selection of $J$.

The various mappings appearing in the following Definition 1.1 have been studied widely and deeply by many authors; see, e.g., [1-11] for more details.

Definition 1.1. Let $C$ be a nonempty subset of a Banach space $E$. A mapping $T: C \rightarrow C$ is called

(i) nonexpansive if

$$
\|T x-T y\| \leq\|x-y\|, \quad \forall x, y \in C
$$

(ii) quasi-nonexpansive if the fixed point set $F(T):=\{x \in C: T x=x\} \neq \emptyset$, and

$$
\left\|T x-x^{*}\right\| \leq\left\|x-x^{*}\right\|, \quad \text { for all } x \in C \text { and } x^{*} \in F(T) ;
$$

(iii) asymptotically nonexpansive if there is a sequence $\left\{k_{n}\right\}_{n \geq 0} \subset[1, \infty)$ with $\lim _{n \rightarrow \infty} k_{n}=$ 1 such that

$$
\left\|T^{n} x-T^{n} y\right\| \leq k_{n}\|x-y\|, \quad \text { for all } x, y \in C \text { and } n \geq 0
$$

(iv) asymptotically quasi-nonexpansive if $F(T) \neq \emptyset$ and there is a sequence $\left\{k_{n}\right\}_{n \geq 0} \subset$ $[1, \infty)$ with $\lim _{n \rightarrow \infty} k_{n}=1$ such that

$$
\left\|T^{n} x-x^{*}\right\| \leq k_{n}\left\|x-x^{*}\right\|, \quad \text { for all } x \in C, x^{*} \in F(T), \text { and } n \geq 0 ;
$$

(v) asymptotically demicontractive if $F(T) \neq \emptyset$, there exist a constant $k \in[0,1)$ and a sequence $\left\{a_{n}\right\}_{n \geq 0}$, and for each $x \in C$ and $x^{*} \in F(T)$ there exists $j\left(x-x^{*}\right) \in J\left(x-x^{*}\right)$ such that

$$
\left\langle\left(I-T^{n}\right) x, j\left(x-x^{*}\right)\right\rangle \geq \frac{1}{2}(1-k)\left\|x-T^{n} x\right\|^{2}-\frac{1}{2}\left(a_{n}^{2}-1\right)\left\|x-x^{*}\right\|^{2} .
$$

(vi) uniformly L-Lipschitzian if there exists a constant $L>0$ such that

$$
\left\|T^{n} x-T^{n} y\right\| \leq L\|x-y\|, \quad \text { for all } x, y \in C \text { and } n \geq 1 .
$$

In Hilbert spaces, the concept of an asymptotically demicontractive mapping has been given very early; see, e.g., $[2,6]$. Indeed, for a nonempty subset $C$ of a Hilbert space, a 
mapping $T: C \rightarrow C$ with $F(T) \neq \emptyset$ is asymptotically demicontractive if and only if there exists a sequence $\left\{a_{n}\right\}_{n=0}^{\infty}$ with $\lim _{n \rightarrow \infty} a_{n}=1$ such that

$$
\left\|T^{n} x-x^{*}\right\|^{2} \leq a_{n}^{2}\left\|x-x^{*}\right\|^{2}+k\left\|x-T^{n} x\right\|^{2}
$$

for some $k \in[0,1)$ and for all $x \in C, x^{*} \in F(T)$ and $n \geq 1$.

In 1973, Petryshyn and Willianson [7] proved a necessary and sufficient condition for the strong convergence of the Picard and the Mann iterative schemes to a fixed point of a quasi-nonexpansive mapping in a Hilbert space. Subsequently, Liu [3, 4] extended the above results and obtained some necessary and sufficient conditions for an Ishikawa-type iterative scheme with errors to converge to a fixed point of an asymptotically quasi-nonexpansive map. Recently, Moore and Nnoli [5] proved necessary and sufficient conditions for the strong convergence of the Mann iteration process to a fixed point of an asymptotically demicontractive map in a real Banach space. Their theorems thus improve and extend the results of Liu [3, 4], Osilike [6] and several others.

Theorem 1.2. ([5, Theorems 3.2 and 3.3]) Let $E$ be a real Banach space. Let $T: E \rightarrow E$ be a uniformly L-Lipschitzian asymptotically demicontractive map with a nonempty fixed point set $F(T)$. Suppose $\left\{a_{n}\right\}_{n \geq 0}$ is the sequence associated to the asymptotic demicontractivity of $T$ and $\left\{c_{n}\right\}_{n \geq 0} \subset[0,1]$ is a sequence such that

$$
\sum_{n \geq 0} c_{n}^{2}<\infty \quad \text { and } \quad \sum_{n \geq 0} c_{n}\left(a_{n}^{2}-1\right)<\infty
$$

Let $\left\{x_{n}\right\}_{n \geq 0}$ be the sequence generated from an arbitrary $x_{0} \in E$ by

$$
x_{n+1}=\left(1-c_{n}\right) x_{n}+c_{n} T^{n} x_{n}, \quad n \geq 0 .
$$

Then $\left\{x_{n}\right\}_{n \geq 0}$ converges strongly to a fixed point of $T$ if and only if $\lim _{\inf _{n \rightarrow \infty}} d\left(x_{n}, F(T)\right)=$ 0 . In particular, $\left\{x_{n}\right\}_{n \geq 0}$ converges strongly to an $x^{*} \in F(T)$ if and only if there exists a subsequence of $\left\{x_{n}\right\}_{n \geq 0}$ converging strongly to $x^{*}$.

In this paper, we introduce a new class of asymptotically demicontractive type mappings in real Banach spaces $E$.

Definition 1.3. Let $C$ be a nonempty subset of $E$ and $S: C \rightarrow C$ be an operator. A mapping $T: C \rightarrow C$ is said to be asymptotically $S$-demicontractive if $F(T) \neq \emptyset$ and there exist real sequences $\left\{a_{n}\right\}_{n \geq 0},\left\{k_{n}\right\}_{n \geq 0} \subset[1, \infty)$ and $\left\{\varepsilon_{n}\right\}_{n \geq 0} \subset[0, \infty)$, and for each $x \in C$ and $x^{*} \in F(T)$ there exists $j\left(x-x^{*}\right) \in J\left(x-x^{*}\right)$ such that

$$
\begin{gathered}
\left\langle\left(I-T^{n}\right) x, j\left(x-x^{*}\right)\right\rangle \geq-\frac{1}{2}\left[\left(k_{n}-1\right)\left\|x-T^{n} x\right\|^{2}+\left(a_{n}^{2}-1\right)\left\|x-x^{*}\right\|^{2}+\varepsilon_{n}\right] \\
-\left\langle T^{n} S y-T^{n} y, j\left(x-x^{*}\right)\right\rangle, \quad \forall y \in C .
\end{gathered}
$$

It is remarkable that if $\varepsilon_{n}=0$ and $k_{n}=k \in[0,1)$ for all $n \geq 0$, and $S=I$ the identity mapping, then the concept of asymptotically $S$-demicontractive mapping reduces to the one of asymptotically demicontractive mapping. 
The following is the main result in this paper, which extends and improves recent result of Moore and Nnoli [5].

Theorem 1.4. Let $C$ be a nonempty closed convex subset of a real Banach space E, let $S: C \rightarrow C$ be a quasi-nonexpansive mapping, and let $T: C \rightarrow C$ be a uniformly L-Lipschitzian asymptotically $S$-demicontractive mapping with sequences $\left\{a_{n}\right\}_{n \geq 0},\left\{k_{n}\right\}_{n \geq 0} \subset[1, \infty)$ and $\left\{\varepsilon_{n}\right\}_{n \geq 0} \subset[0, \infty)$. Suppose the common fixed point set $F:=F(T) \cap S(T) \neq \emptyset$, and there is a real sequence $\left\{c_{n}\right\}_{n \geq 0} \subset[0,1]$ satisfying that

$$
\sum_{n \geq 0} c_{n}^{2}<\infty, \quad \sum_{n \geq 0} c_{n}\left(a_{n}^{2}-1\right)<\infty, \quad \sum_{n \geq 0} c_{n}\left(k_{n}-1\right)<\infty, \quad \text { and } \sum_{n \geq 0} c_{n} \varepsilon_{n}<\infty .
$$

Let $\left\{x_{n}\right\}_{n \geq 0}$ be the sequence generated from an arbitrary $x_{0} \in C$ by

$$
x_{n+1}=\left(1-c_{n}\right) S x_{n}+c_{n} T^{n} x_{n}, \quad n \geq 0 .
$$

Then $\left\{x_{n}\right\}_{n \geq 0}$ converges strongly to an element of $F$ if and only if $\liminf _{n \rightarrow \infty} d\left(x_{n}, F\right)=0$. In particular, $\left\{x_{n}\right\}_{n \geq 0}$ converges strongly to $x^{*} \in F$ if and only if there exists an infinite subsequence of $\left\{x_{n}\right\}_{n \geq 0}$ which converges strongly to $x^{*} \in F$.

\section{The proofs}

In the sequel we shall make use of the following lemmas.

Lemma 2.1. (Tan and Xu [10, Lemma 1, p. 303]) Let $\left\{\beta_{n}\right\}_{n \geq 0}$ and $\left\{b_{n}\right\}_{n \geq 0}$ be sequences of nonnegative real numbers satisfying the inequality

$$
\beta_{n+1} \leq \beta_{n}+b_{n}, \quad n \geq 0 .
$$

If $\sum_{n \geq 0} b_{n}<\infty$ then $\lim _{n \rightarrow \infty} \beta_{n}$ exists.

Lemma 2.2. (Chang [1, Lemma 1.1, p. 847]) Let $E$ be a real normed linear space. Then the following inequality holds:

$$
\|x+y\|^{2} \leq\|x\|^{2}+2\langle y, j(x+y)\rangle, \quad \text { for all } x, y \in E \text { and } j(x+y) \in J(x+y) .
$$

Lemma 2.3. Assuming the conditions stated in Theorem 1.4, we have for each $x^{*} \in F$ and $n, m \geq 1$,

(a) there exists $M>0$ such that $\left\|x_{n}-x^{*}\right\| \leq M$,

(b) $\lim _{n \rightarrow \infty}\left\|x_{n}-x^{*}\right\|$ exists,

(c) $\left\|x_{n+1}-x^{*}\right\|^{2} \leq\left(1+c_{n}^{2}\right)\left\|x_{n}-x^{*}\right\|^{2}+\mu_{n}$ for some $\left\{\mu_{n}\right\}_{n \geq 0}$ with $\sum_{n \geq 0} \mu_{n}<\infty$, 
(d) $\left\|x_{n+m}-x^{*}\right\|^{2} \leq D\left\|x_{n}-x^{*}\right\|^{2}+D \sum_{i \geq 0} \mu_{i}$, where $D=e^{\sum_{i \geq 0} c_{i}^{2}}$.

Proof of (a) and (b). From (2), (3) and Definition $1.1(\mathrm{v})$ with $y=x_{n}$ we obtain

$$
\begin{aligned}
\left\|x_{n+1}-x^{*}\right\|^{2} \leq & \left(1-c_{n}\right)^{2}\left\|S x_{n}-x^{*}\right\|^{2}+2 c_{n}\left\langle T^{n} x_{n}-x^{*}, j\left(x_{n+1}-x^{*}\right)\right\rangle \\
\leq & \left(1-c_{n}\right)^{2}\left\|x_{n}-x^{*}\right\|^{2}+2 c_{n}\left\langle T^{n} x_{n}-x^{*}, j\left(x_{n+1}-x^{*}\right)\right\rangle \\
= & \left(1-c_{n}\right)^{2}\left\|x_{n}-x^{*}\right\|^{2}-2 c_{n}\left\langle x_{n+1}-T^{n} x_{n+1}, j\left(x_{n+1}-x^{*}\right)\right\rangle \\
& +2 c_{n}\left\langle x_{n+1}-x^{*}, j\left(x_{n+1}-x^{*}\right)\right\rangle+2 c_{n}\left\langle T^{n} x_{n}-T^{n} x_{n+1}, j\left(x_{n+1}-x^{*}\right)\right\rangle \\
\leq & \left(1-c_{n}\right)^{2}\left\|x_{n}-x^{*}\right\|^{2}+c_{n}\left(k_{n}-1\right)\left\|x_{n+1}-T^{n} x_{n+1}\right\|^{2} \\
& +c_{n}\left(a_{n}^{2}-1\right)\left\|x_{n+1}-x^{*}\right\|^{2}+2 c_{n}\left\langle T^{n} S x_{n}-T^{n} x_{n}, j\left(x_{n+1}-x^{*}\right)\right\rangle+c_{n} \varepsilon_{n} \\
& +2 c_{n}\left\|x_{n+1}-x^{*}\right\|^{2}+2 c_{n}\left\langle T^{n} x_{n}-T^{n} x_{n+1}, j\left(x_{n+1}-x^{*}\right)\right\rangle \\
= & \left(1-c_{n}\right)^{2}\left\|x_{n}-x^{*}\right\|^{2}+c_{n}\left(k_{n}-1\right)\left\|x_{n+1}-T^{n} x_{n+1}\right\|^{2} \\
& +c_{n}\left(a_{n}^{2}-1\right)\left\|x_{n+1}-x^{*}\right\|^{2}+2 c_{n}\left\|x_{n+1}-x^{*}\right\|^{2} \\
& +2 c_{n}\left\langle T^{n} S x_{n}-T^{n} x_{n+1}, j\left(x_{n+1}-x^{*}\right)\right\rangle+c_{n} \varepsilon_{n} \\
\leq & \left(1-c_{n}\right)^{2}\left\|x_{n}-x^{*}\right\|^{2}+c_{n}\left(k_{n}-1\right)\left\|x_{n+1}-T^{n} x_{n+1}\right\|^{2} \\
& +c_{n}\left(a_{n}^{2}-1\right)\left\|x_{n+1}-x^{*}\right\|^{2}+2 c_{n}\left\|x_{n+1}-x^{*}\right\|^{2} \\
& +2 c_{n}\left\|T^{n} S x_{n}-T^{n} x_{n+1}\right\|\left\|x_{n+1}-x^{*}\right\|+c_{n} \varepsilon_{n} .
\end{aligned}
$$

Observe that

$$
\begin{aligned}
&\left\|x_{n+1}-x^{*}\right\| \leq\left(1-c_{n}\right)\left\|S x_{n}-x^{*}\right\|+c_{n}\left\|T^{n} x_{n}-x^{*}\right\| \\
& \leq\left(1-c_{n}\right)\left\|x_{n}-x^{*}\right\|+c_{n} L\left\|x_{n}-x^{*}\right\| \\
& \leq\left(1+L c_{n}\right)\left\|x_{n}-x^{*}\right\|, \\
&\left\|x_{n+1}-T^{n} x_{n+1}\right\| \leq\left\|x_{n+1}-x^{*}\right\|+\left\|T^{n} x_{n+1}-x^{*}\right\| \\
& \leq(1+L)\left\|x_{n+1}-x^{*}\right\| \\
& \leq(1+L)\left(1+L c_{n}\right)\left\|x_{n}-x^{*}\right\| \\
& \leq(1+L)^{2}\left\|x_{n}-x^{*}\right\|,
\end{aligned}
$$

and

$$
\begin{aligned}
\left\|T^{n} S x_{n}-T^{n} x_{n+1}\right\| & \leq L\left\|S x_{n}-x_{n+1}\right\|=L c_{n}\left\|T^{n} x_{n}-S x_{n}\right\| \\
& \leq L c_{n}\left(\left\|T^{n} x_{n}-x^{*}\right\|+\left\|S x_{n}-x^{*}\right\|\right) \\
& \leq c_{n} L(1+L)\left\|x_{n}-x^{*}\right\| \\
& \leq c_{n}(1+L)^{2}\left\|x_{n}-x^{*}\right\| .
\end{aligned}
$$

Substituting (5)-(7) in (4), we have

$$
\begin{aligned}
\left\|x_{n+1}-x^{*}\right\|^{2} \leq & \left(1-c_{n}\right)^{2}\left\|x_{n}-x^{*}\right\|^{2}+c_{n}\left(k_{n}-1\right)(1+L)^{4}\left\|x_{n}-x^{*}\right\|^{2} \\
& +c_{n}\left(a_{n}^{2}-1\right)(1+L)^{2}\left\|x_{n}-x^{*}\right\|^{2}+2 c_{n}\left(1+L c_{n}\right)^{2}\left\|x_{n}-x^{*}\right\|^{2} \\
& +2 c_{n}^{2}(1+L)^{3}\left\|x_{n}-x^{*}\right\|^{2}+c_{n} \varepsilon_{n} \\
= & \left(1+\gamma_{n}\right)\left\|x_{n}-x^{*}\right\|^{2}+c_{n} \varepsilon_{n},
\end{aligned}
$$

where $\gamma_{n}=c_{n}\left(k_{n}-1\right)(1+L)^{4}+c_{n}\left(a_{n}^{2}-1\right)(1+L)^{2}+c_{n}^{2}\left[1+2\left(2 L+L^{2} c_{n}\right)+2(1+L)^{3}\right]$. According to the conditions that $\sum_{n \geq 0} c_{n}^{2}<\infty, \sum_{n \geq 0} c_{n}\left(a_{n}^{2}-1\right)<\infty$ and $\sum_{n \geq 0} c_{n}\left(k_{n}-1\right)<\infty$, we know 
that $\sum_{n \geq 0} \gamma_{n}<\infty$. From (8) we obtain

$$
\begin{aligned}
\left\|x_{n+1}-x^{*}\right\|^{2} \leq & \left(1+\gamma_{n}\right)\left(1+\gamma_{n-1}\right)\left\|x_{n-1}-x^{*}\right\|^{2}+\left(1+\gamma_{n}\right) c_{n-1} \varepsilon_{n-1}+c_{n} \varepsilon_{n} \\
\leq & \left(1+\gamma_{n}\right)\left(1+\gamma_{n-1}\right)\left(1+\gamma_{n-2}\right)\left\|x_{n-2}-x^{*}\right\|^{2} \\
& +\left(1+\gamma_{n}\right)\left(1+\gamma_{n-1}\right) c_{n-2} \varepsilon_{n-2}+\left(1+\gamma_{n}\right) c_{n-1} \varepsilon_{n-1}+c_{n} \varepsilon_{n} \\
\vdots & \\
\leq & \prod_{i=0}^{n}\left(1+\gamma_{i}\right)\left\|x_{0}-x^{*}\right\|^{2}+\sum_{j=0}^{n-1} c_{j} \varepsilon_{j} \prod_{i=j+1}^{n}\left(1+\gamma_{i}\right)+c_{n} \varepsilon_{n} \\
\leq & \prod_{i=0}^{n}\left(1+\gamma_{i}\right)\left\|x_{0}-x^{*}\right\|^{2}+\prod_{i=0}^{n}\left(1+\gamma_{i}\right) \sum_{j=0}^{n} c_{j} \varepsilon_{j} \\
\leq & e^{\sum_{i \geq 0}^{n} \gamma_{i}}\left\|x_{0}-x^{*}\right\|^{2}+e^{\sum_{i \geq 0} \gamma_{i}} \cdot \sum_{j \geq 0} c_{j} \varepsilon_{j},
\end{aligned}
$$

and hence $\left\|x_{n}-x^{*}\right\| \leq M$ for some $M>0$. If we set $\beta_{n}=\left\|x_{n}-x^{*}\right\|^{2}$ and $b_{n}=\gamma_{n} M^{2}+c_{n} \varepsilon_{n}$ then, by Lemma $2.1, \lim _{n \rightarrow \infty}\left\|x_{n}-x^{*}\right\|$ exists.

Proof of (c). From (8) we get

$$
\left\|x_{n+1}-x^{*}\right\|^{2} \leq\left[1+c_{n}^{2}+\lambda_{n}\right]\left\|x_{n}-x^{*}\right\|^{2}+c_{n} \varepsilon_{n}
$$

where $\lambda_{n}=\gamma_{n}-c_{n}^{2}=c_{n}\left(k_{n}-1\right)(1+L)^{4}+c_{n}\left(a_{n}^{2}-1\right)(1+L)^{2}+2 c_{n}^{2}\left[\left(2 L+L^{2} c_{n}\right)+(1+L)^{3}\right]$. Moreover,

$$
\begin{aligned}
\left\|x_{n+1}-x^{*}\right\|^{2} & \leq\left[1+c_{n}^{2}+\lambda_{n}\right]\left\|x_{n}-x^{*}\right\|^{2}+c_{n} \varepsilon_{n} \\
& \leq\left(1+c_{n}^{2}\right)\left\|x_{n}-x^{*}\right\|^{2}+\lambda_{n} M^{2}+c_{n} \varepsilon_{n} \\
& =\left(1+c_{n}^{2}\right)\left\|x_{n}-x^{*}\right\|^{2}+\mu_{n},
\end{aligned}
$$

where $\mu_{n}=\lambda_{n} M^{2}+c_{n} \varepsilon_{n}=\left(\gamma_{n}-c_{n}^{2}\right) M^{2}+c_{n} \varepsilon_{n}$. Observe that $\sum_{n \geq 0} \mu_{n}<\infty$.

Proof of (d). From (c) we obtain for each $n, m \geq 1$,

$$
\begin{aligned}
& \left\|x_{n+m}-x^{*}\right\|^{2} \leq\left(1+c_{n+m-1}^{2}\right)\left\|x_{n+m-1}-x^{*}\right\|^{2}+\mu_{n+m-1} \\
& \leq\left(1+c_{n+m-1}^{2}\right)\left(1+c_{n+m-2}^{2}\right)\left\|x_{n+m-2}-x^{*}\right\|^{2}+\left(1+c_{n+m-1}^{2}\right) \mu_{n+m-2}+\mu_{n+m-1} \\
& \leq\left(1+c_{n+m-1}^{2}\right)\left(1+c_{n+m-2}^{2}\right)\left(1+c_{n+m-3}^{2}\right)\left\|x_{n+m-3}-x^{*}\right\|^{2} \\
& +\left(1+c_{n+m-1}^{2}\right)\left(1+c_{n+m-2}^{2}\right) \mu_{n+m-3}+\left(1+c_{n+m-1}^{2}\right) \mu_{n+m-2}+\mu_{n+m-1} \\
& \vdots \\
& \leq \prod_{i=n}^{n+m-1}\left(1+c_{i}^{2}\right)\left\|x_{n}-x^{*}\right\|^{2}+\prod_{i=n}^{n+m-1}\left(1+c_{i}^{2}\right) \sum_{i=n}^{n+m-1} \mu_{i} \\
& \leq e^{\sum_{i=n}^{n+m-1} c_{i}^{2}}\left\|x_{n}-x^{*}\right\|^{2}+e^{\sum_{i=n}^{n+m-1} c_{i}^{2}} \sum_{i=n}^{n+m-1} \mu_{i} \\
& \leq D\left\|x_{n}-x^{*}\right\|^{2}+D \sum_{i \geq 0} \mu_{i}
\end{aligned}
$$

where $D=e^{\sum_{i \geq 0} c_{i}^{2}}$. This completes the proof. 
Proof of Theorem 1.4. From Lemma 2.3 (c) we obtain

$$
\left[d\left(x_{n+1}, F\right)\right]^{2} \leq\left(1+c_{n}^{2}\right)\left[d\left(x_{n}, F\right)\right]^{2}+\mu_{n}
$$

where $F:=F(T) \cap S(T) \neq \emptyset$ and $\sum_{n \geq 0} \mu_{n}<\infty$. Since $\liminf _{n \rightarrow \infty} d\left(x_{n}, F\right)=0$, from Lemma $2.3(\mathrm{~d})$ we derive the boundedness of $\left\{d\left(x_{n}, F\right)\right\}$. Hence it follows from Lemma 2.1 that

$$
\lim _{n \rightarrow \infty} d\left(x_{n}, F\right)=0 .
$$

It now suffices to show that $\left\{x_{n}\right\}$ is a Cauchy sequence in $C$. Indeed, put $\tau=\prod_{i=0}^{\infty}\left(1+c_{i}^{2}\right)$. Then $1 \leq \tau<\infty$. Since $\lim _{n \rightarrow \infty} d\left(x_{n}, F\right)=0$ and $\sum_{n \geq 0} \mu_{n}<\infty$, for arbitrarily given $\epsilon>0$ there exists a positive integer $N_{1}$ such that for all $n \geq N_{1}$

$$
d\left(x_{n}, F\right)<\frac{\epsilon}{\sqrt{8 \tau}} \text { and } \sum_{i=n}^{\infty} \mu_{i}<\frac{\epsilon^{2}}{8 \tau} .
$$

In particular there exists $\hat{x} \in F$ such that $d\left(x_{N_{1}}, \hat{x}\right)<\epsilon / \sqrt{8 \tau}$. Now from Lemma 2.3 (c) we conclude that

$$
\begin{aligned}
\left\|x_{n}-\hat{x}\right\|^{2} & \leq\left(1+c_{n-1}^{2}\right)\left\|x_{n-1}-\hat{x}\right\|^{2}+\mu_{n-1} \\
& \leq\left(1+c_{n-1}^{2}\right)\left(1+c_{n-2}^{2}\right)\left\|x_{n-2}-\hat{x}\right\|^{2}+\left(1+c_{n-1}^{2}\right) \mu_{n-2}+\mu_{n-1} \\
& \vdots \\
& \leq \prod_{i=N_{1}}^{n-1}\left(1+c_{i}^{2}\right)\left\|x_{N_{1}}-\hat{x}\right\|^{2}+\sum_{j=N_{1}}^{n-2} \mu_{j} \prod_{i=j+1}^{n-1}\left(1+c_{i}^{2}\right)+\mu_{n-1} \\
& \leq \prod_{i=N_{1}}^{n-1}\left(1+c_{i}^{2}\right)\left[\left\|x_{N_{1}}-\hat{x}\right\|^{2}+\sum_{j=N_{1}}^{n-1} \mu_{j}\right] \\
& \leq \tau\left[\left\|x_{N_{1}}-\hat{x}\right\|^{2}+\sum_{j=N_{1}}^{n-1} \mu_{j}\right] \\
& \leq \tau\left[\left\|x_{N_{1}}-\hat{x}\right\|^{2}+\sum_{j=N_{1}}^{\infty} \mu_{j}\right] \\
& \leq \tau\left[\frac{\epsilon^{2}}{8 \tau}+\frac{\epsilon^{2}}{8 \tau}\right]=\frac{\epsilon^{2}}{4}
\end{aligned}
$$

for all $n \geq N_{1}$. Consequently, we deduce that for all $n \geq N_{1}$ and $m \geq 1$

$$
\left\|x_{n+m}-x_{n}\right\|^{2} \leq 2\left\|x_{n+m}-\hat{x}\right\|^{2}+2\left\|x_{n}-\hat{x}\right\|^{2} \leq 2 \cdot \frac{\epsilon^{2}}{4}+2 \cdot \frac{\epsilon^{2}}{4}=\epsilon^{2},
$$

and hence $\left\|x_{n+m}-x_{n}\right\| \leq \epsilon$. Thus, $\lim _{n \rightarrow \infty} x_{n}$ exists due to the completeness of $E$. Note that $C$ is closed. We may suppose that $\lim _{n \rightarrow \infty} x_{n}=\bar{x} \in C$. We now show that $\bar{x} \in F$. Indeed, for arbitrarily given $\bar{\epsilon}>0$ there exists a positive integer $N_{2} \geq N_{1}$ such that for all $n \geq N_{2}$

$$
\left\|x_{n}-\bar{x}\right\|<\frac{\bar{\epsilon}}{2(1+L)} \quad \text { and } \quad d\left(x_{n}, F\right)<\frac{\bar{\epsilon}}{2(1+L)} .
$$


Thus, there exists $y^{*} \in F$ such that

$$
\left\|x_{N_{2}}-y^{*}\right\|=d\left(x_{N_{2}}, y^{*}\right)<\frac{\bar{\epsilon}}{2(1+L)} .
$$

We then have the following estimates:

$$
\begin{aligned}
\|T \bar{x}-\bar{x}\| & \leq\left\|T \bar{x}-T x_{N_{2}}\right\|+\left\|T x_{N_{2}}-y^{*}\right\|+\left\|y^{*}-x_{N_{2}}\right\|+\left\|x_{N_{2}}-\bar{x}\right\| \\
& \leq(1+L)\left\|x_{N_{2}}-\bar{x}\right\|+(1+L)\left\|x_{N_{2}}-y^{*}\right\| \\
& \leq(1+L) \cdot \frac{\bar{\epsilon}}{2(1+L)}+(1+L) \cdot \frac{\bar{\epsilon}}{2(1+L)}=\bar{\epsilon}
\end{aligned}
$$

and

$$
\begin{aligned}
\|S \bar{x}-\bar{x}\| & \leq\left\|S \bar{x}-S x_{N_{2}}\right\|+\left\|S x_{N_{2}}-y^{*}\right\|+\left\|y^{*}-x_{N_{2}}\right\|+\left\|x_{N_{2}}-\bar{x}\right\| \\
& \leq 2\left\|x_{N_{2}}-\bar{x}\right\|+2\left\|x_{N_{2}}-y^{*}\right\| \\
& \leq 2 \cdot \frac{\bar{\epsilon}}{2(1+L)}+2 \cdot \frac{\bar{\epsilon}}{2(1+L)}=\frac{2 \bar{\epsilon}}{1+L} .
\end{aligned}
$$

Since $\bar{\epsilon}>0$ is arbitrary, we infer that $T \bar{x}=\bar{x}$ and $S \bar{x}=\bar{x}$. This completes the proof.

\section{References}

[1] S. S. Chang, Some results for asymptotically pseudo-contractive mappings and asymptotically nonexpansive mappings, Proc. Amer. Math. Soc., 129 (2001), no. 3, 845-853.

[2] Q. H. Liu, On Naimpally and Singh's open questions, J. Math. Anal. Appl., 124 (1987), no. $1,157-164$.

[3] Q. H. Liu, Iterative sequences for asymptotically quasi-nonexpansive mappings, J. Math. Anal. Appl., 259 (2001), no. 1, 1-7.

[4] Q. H. Liu, Iterative sequences for asymptotically quasi-nonexpansive mappings with error members, J. Math. Anal. Appl., 259 (2001), no. 1, 18-24.

[5] C. Moore and B. V. C. Nnoli, Iterative sequence for asymptotically demicontractive maps in Banach spaces, J. Math. Anal. Appl., 302 (2005), no. 2, 557-562.

[6] M. O. Osilike, Iterative approximation of fixed points of asymptotically demicontractive mappings, Indian J. Pure Appl. Math., 29 (1998), no. 12, 1291-1300.

[7] W. V. Petryshyn and T. E. Williamson, Jr., Strong and weak convergence of successive approximations for quasi-nonexpansive mappings, J. Math. Anal. Appl. 43 (1973), 459 497.

[8] B. E. Rhoades, Comments on two fixed point iterative methods, J. Math. Anal. Appl., 56 (1976), 741-750.

[9] J. Schu, Iterative construction of fixed points of asymptotically nonexpansive mappings, J. Math. Anal. Appl., 158 (1991), no. 2, 407-413. 
[10] K. K. Tan and H. K. Xu, Approximating fixed points of nonexpansive mappings by the Ishikawa iteration process, J. Math. Anal. Appl., 178 (1993), no. 2, 301-308.

[11] K. K. Tan and H. K. Xu, Fixed point iteration processes for asymptotically nonexpansive mappings, Proc. Amer. Math. Soc., 122 (1994), no. 3, 733-739. 